\title{
ISOLATION AND CHARACTERIZATION OF FURANOMYCIN NONPRODUCING Streptomyces threomyceticus MUTANTS
}

\author{
Ronald J. PARry and Naiquan YANG \\ Department of Chemistry, Rice University, \\ P.O. Box 1892, Houston, TX 77251, U.S.A.
}

(Received for publication January 27, 1992)

\begin{abstract}
Stable mutants that are blocked in the production of the polyketide antibiotic furanomycin were generated by treatment of Streptomyces threomyceticus (ATCC 15795) with a combination of $N$-methyl- $N^{\prime}$-nitro- $N$-nitrosoguanidine (NTG) and ultraviolet light. On the basis of their cosynthetic properties in mixed culture, the mutants were grouped into eleven phenotypic classes. The polarity of the cosynthetic reactions for seven classes of mutants was revealed by filtrate feeding experiments. This allowed them to be arranged in the most probable linear sequence of metabolic blocks. One of the remaining groups of mutants exhibited no cosynthetic behavior and is believed to be blocked in a gene that regulates furanomycin biosynthesis.
\end{abstract}

In 1967 KATAGIRI et al. reported ${ }^{1)}$ that the fermentation broth of Streptomyces threomyceticus (ATCC 15795) contains the novel antibiotic furanomycin (1) (Fig. 1). The compound was found to be a competitive antagonist of $\mathrm{L}$-isoleucine and to inhibit the growth of T-even coliphage. Recently, furanomycin has been shown to be charged to isoleucine tRNA by isoleucyl-tRNA synthetase from Escherichia coli and to be subsequently incorporated into protein. ${ }^{2)}$ Previous investigations in our laboratory ${ }^{3)}$ have demonstrated that $\mathbf{1}$ is a polyketide derived from two acetate units and one propionate unit, with the latter serving as the starter unit. Furthermore, the ether oxygen atom of furanomycin was found to be derived from molecular oxygen and to be introduced at $\mathrm{C}-2$ of the propionate unit with loss of the 2 pro- $R$ hydrogen atom and overall inversion of configuration. ${ }^{4)}$ These observations and the known stereochemistry of allylic rearrangements catalyzed by $\beta$-hydroxydecanoylthioester dehydrase ${ }^{5)}$ led to the postulate that the biosynthesis of furanomycin proceeds by epoxidation of trans, cis-2,3,5,6-heptadienoic acid (2) to a diepoxide (3) which could then undergo ring-opening of the 5,6-epoxide with subsequent ring-closure to yield the $\alpha$-hydroxy acid 4 (Fig. 1). The $\alpha$-hydroxyacid could then be converted into furanomycin by oxidation

Fig. 1. The proposed biosynthetic pathway to furanomycin.

$$
\begin{aligned}
& \mathrm{CH}_{3} \mathrm{CH}_{2} \mathrm{COOH} \\
& 2 \mathrm{CH}_{3} \mathrm{COOH}
\end{aligned}
$$

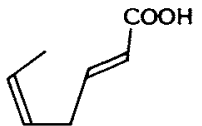

2

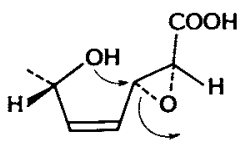

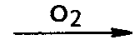<smiles>CCC12CCC(C3OC3C(=O)O)C1(CC)O2</smiles>

3<smiles>C[C@H]1C=C[C@H]([C@H](N)C(=O)O)O1</smiles> 
of the hydroxyl group to give an $\alpha$-keto acid followed by transamination. The mechanism postulated for the formation of furanomycin is of particular interest, since it bears a close resemblance to that suggested for the biosynthesis of the polyether antibiotics. ${ }^{6)}$ With the eventual goal of gaining additional insight into the biosynthesis of furanomycin, we have therefore prepared and classified furanomycin-nonproducing mutants (Fur ${ }^{-}$) of S. threomyceticus.

\section{Materials and Methods}

\section{Bacterial Strains}

The strains of $\bar{S}$. threomyceticus used in this work are listed in Table 1. S. threomyceticus ATCC 15795 was obtained from the American Type Culture Collection, Rockville, MD, and Bacillus subtilis UC-28 was a gift from The Upjohn Company, Kalamazoo, MI.

\section{Growth Media}

S. threomyceticus was maintained on BENNETT's agar which contained the following components per liter: yeast extract (Difco Laboratories, Detroit, MI), 1 g; beef extract (Difco), 1 g; N-Z-amine type A (Sheffield products, Kraft Inc., Woods Corners, Norwich, NY), 2 g; glucose, 10 g; and Bacto agar (Difco), $15 \mathrm{~g}$. The antibiotic production medium used for the isolation of furanomycin-nonproducing mutants and for cosynthesis experiments contained the following ingredients per liter: starch, $20 \mathrm{~g}$; soybean meal, $10 \mathrm{~g}$; corn steep liquor (Corn Products, Unit of CPC North America, Argo, IL), $5 \mathrm{~g} ; \mathrm{NaCl}, 5 \mathrm{~g} ; \mathrm{CaCO}_{3}, 3.5 \mathrm{~g}$. The medium used in bioassay and bioautography was the glucose-inorganic salts medium described by KATAGIRI et al. ${ }^{1)}$ The Fur ${ }^{-}$mutants were evaluated for furanomycin resistance on the minimal medium (MM) described by Hopwoon et al. ${ }^{7)}$ All these media were adjusted to $\mathrm{pH} 7.0$ before sterilization. Brain-heart infusion medium (Difco) with $1.5 \%$ agar was used for the growth of $B$. subtilis UC-28.

Isolation of Furanomycin-nonproducing Mutants

For the isolation of furanomycin-nonproducing mutants ( $\mathrm{Fur}^{-}$), mutagenesis was carried out by treatment with $N$-methyl- $N^{\prime}$-nitro- $N$-nitrosoguanidine (NTG; Aldrich Chemical Company, Inc., Milwaukee, WI) according to the method of DELIC et al. ${ }^{8)}$ in combination with UV light from a germicidal mercury lamp. In order to create the mutations, a killing rate from 99.0 to $99.999 \%$ was used. Approximately $50 \%$ of the colonies surviving mutagenesis were observed to possess normal morphology. Colonies grown from single spores and with normal morphology were transferred to duplicate BENNETT's agar plates, and incubated at $30^{\circ} \mathrm{C}$ for 8 days. One colony of the pair was then transferred to a $16 \times 150 \mathrm{~mm}$ tube containing $2.5 \mathrm{ml}$ of antibiotic production medium and the tube shaken at $30^{\circ} \mathrm{C}, 250 \mathrm{rpm}$ for 7 days. If the bioassay of the fermentation filtrate from the tube showed a lack of furanomycin production, then the duplicate colony was used to inoculate a BENNETT's agar slant. After sporulation the organism on the slant was re-evaluated for a lack of furanomycin production in tube fermentation. All of the slants which showed a lack of furanomycin production were then used to prepare spore suspensions in $20 \%$ glycerol which were stored at $-80^{\circ} \mathrm{C}$. The stability of the Fur ${ }^{-}$phenotype was then evaluated for each spore suspension through three serial transfers on BENNETT's agar. Only those mutants that exhibited stability of the Fur ${ }^{-}$ phenotype were selected for further study.

Evaluation of Furanomycin Resistance of $\mathrm{Fur}^{-}$Mutants

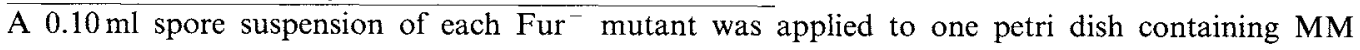
medium. A $12.7-\mathrm{mm}$ paper disc impregnated with $100 \mu \mathrm{g}$ of furanomycin was then placed on the surface of the agar and the dish was incubated at $30^{\circ} \mathrm{C}$ for three days. No inhibition zone appeared with any of the mutants. Three non-furanomycin producing strains of Streptomyces (S. lividans TK24, S. ambofaciens (ATCC 15154), and S. griseofuscus (ATCC 23916)) all gave sizeable inhibition zones under these conditions.

Cosynthesis

(1) Mixed-cuitures. A small fragment of mycelium was transferred from slants of two different 
mutants into a tube containing $2 \mathrm{ml}$ of production medium, and the tube was incubated as described above. Bioassay for furanomycin production in the mixed culture was carried out as described under "bioassay."

(2) Precursor feeding. fur mutant type strains were grown in $2 \mathrm{ml}$ of antibiotic production medium for 2 days. The filtrate $(0.50 \mathrm{ml})$ prepared from a culture of another class of fur mutant was added, and the culture to which the filtrate had been administered was then incubated for another 5 days. The culture broth of the fed culture was then bioassayed for furanomycin production. The filtrates were prepared from $30 \mathrm{ml}$ cultures of fur mutant type strains which had been grown for 4 days, centrifuged at $10,000 \mathrm{rpm}$ for 20 minutes, and filtered through a $0.2 \mu \mathrm{m}$ sterile filter (Gelman Sciences Inc., Ann Arbor, MI).

\section{Paper Chromatography and Bioautography}

Strips of Whatman No. 1 paper $(0.7 \mathrm{~cm} \times 20 \mathrm{~cm})$ were used, with the origin being $3 \mathrm{~cm}$ from the lower end of the chromatogram. The solvent system used for development was $n$-butanol - acetic acid - water $(2: 1: 1)$. The fermentation filtrate $(20 \mu \mathrm{l})$ was applied to the origin in $2 \mu \mathrm{l}$ aliquots and the water allowed to evaporate between applications. Development was halted when the solvent front reached a distance of $15 \mathrm{~cm}$ from the origin. After drying in air for 30 minutes, the chromatogram was placed on top of a bioassay dish containing $100 \mathrm{ml}$ of glucose - inorganic salts medium seeded with $0.5 \mathrm{ml}$ of a spore suspension of B. subtilis UC-28 prepared by washing one brain heart infusion agar slant with $2.5 \mathrm{ml}$ of sterile $0.9 \%$ saline. After 30 minutes the paper strip was removed, and the bioassay dish was incubated overnight at $30^{\circ} \mathrm{C}$. Compounds possessing antibiotic activity against $B$. subtilis UC- 28 were identified by the appearance of an inhibition zone.

\section{Bioassay}

The fermentation filtrate $(20 \mu \mathrm{l})$ was applied to a $12.7-\mathrm{mm}$ paper disc (Schleicher \& Schuell, Inc., Keene, $\mathrm{NH}$ ) and the solution allowed to dry in air. The paper disc was then heated at $85^{\circ} \mathrm{C}$ in an oven for 4 hours. After heating, the paper disc was cooled to room temperature and then placed upon the surface of a bioassay dish. The bioassay dish was incubated overnight at $30^{\circ} \mathrm{C}$. The presence of furanomycin was indicated by an inhibition zone. Double layered bioassay plates were found to provide more reproducible results since the interior surface of the glass bioassay dish was uneven. When double layered assay plates were used, the bottom layer consisted of $100 \mathrm{ml}$ of $1.3 \%$ agar which was allowed to solidify before addition of the top layer which was composed of $100 \mathrm{ml}$ of the bioassay medium described above.

\section{Results}

Isolation of Furanomycin-nonproducing Mutants

The results of bioautography showed that the wild type strain of Streptomyces threomyceticus produced two substances in both liquid and solid media which exhibited antibiotic activity against $B$. subtilis UC-28. One of these was furanomycin, while the identity of the other was unknown. Attempts to find a medium which led to the production of only furanomycin were unsuccessful, as were efforts to develop a strain of B. subtilis that lacked sensitivity to the unknown antibiotic. Fortunately, it was discovered that the unknown antibiotic is relatively unstable in comparison to furanomycin. Heating the impregnated assay discs for 4 hours at $85^{\circ} \mathrm{C}$ led to complete loss of the antibiotic activity due to the unknown, while the activity due to furanomycin was unchanged. Therefore, heat treatment was incorporated into the paper disc bioassay procedure used to screen $S$. threomyceticus mutants. Utilizing this method 2,222 colonies were screened, and after three serial transfers on BENNETT's agar slants, $19 \mathrm{Fur}^{-}$mutants were recovered that exhibited a stable Fur $^{-}$phenotype (Table 1).

\section{Classification of $\mathrm{Fur}^{-}$Mutants}

All $19 \mathrm{Fur}^{-}$mutants were tested for their cosynthetic ability by the mixed culture method, while only the type strains were tested for their biotransformation ability using the precursor feeding method. It was 
Table 1. S. threomyceticus strains used in this study.

\begin{tabular}{|c|c|c|c|c|c|}
\hline Strain $^{a}$ & $\begin{array}{l}\text { Production of } \\
\text { furanomycin }\end{array}$ & Genotype & Strain $^{\mathrm{a}}$ & $\begin{array}{l}\text { Production of } \\
\text { furanomycin }\end{array}$ & Genotype \\
\hline ST.4-64 ${ }^{\mathrm{b}}$ & no & fur $A 1$ & ST. $18-67^{b}$ & no & furE 11 \\
\hline ST.4-65 & no & fur $A 2$ & ST.29-3 $3^{b}$ & leaky & fur $F 12$ \\
\hline ST.4-70 & no & fur $A 3$ & ST. $30-52^{\mathrm{b}}$ & no & furG 13 \\
\hline ST.15-65 & no & fur $A 4$ & ST.1-26 & no & fur $H 14$ \\
\hline ST.22-80 & no & fur $A 5$ & ST.2-11 & no & furI 15 \\
\hline ST.13-49b & no & fur $B 6$ & ST.19-20 & no & furI 16 \\
\hline ST.15-63 & no & fur $B 7$ & ST.19-46 & no & furI 17 \\
\hline ST. $15-52^{b}$ & leaky & fur $C 8$ & ST.20-23 & no & furJ 18 \\
\hline ST. $18-15^{\mathrm{b}}$ & leaky & furD 9 & ST.22-13 & no & furK 19 \\
\hline ST.24-24 & leaky & furD 10 & & & \\
\hline
\end{tabular}

a The parent strain is ST.0.

b Type strain used in cosynthesis experiments.

Table 2. Cosynthetic pattern by mixed culture method.

\begin{tabular}{|c|c|c|c|c|c|c|c|c|c|c|c|c|}
\hline $\begin{array}{c}\text { Mutant } \\
\text { class }\end{array}$ & $\begin{array}{l}\text { No. of } \\
\text { mutant } \\
\text { strains }\end{array}$ & I & II & III & IV & $\mathrm{V}$ & VI & VII & VIII & IX & $\mathrm{X}$ & $\mathrm{XI}$ \\
\hline I & 5 & & + & + & + & + & + & + & + & + & - & - \\
\hline II & 2 & & & + & + & + & + & + & - & - & - & - \\
\hline III & 1 & & & & + & + & + & + & - & - & + & - \\
\hline IV & 2 & & & & & + & + & + & - & - & - & - \\
\hline $\mathrm{V}$ & 1 & & & & & & + & + & + & - & - & - \\
\hline VI & 1 & & & & & & & + & - & - & + & - \\
\hline VII & 1 & & & & & & & & - & - & - & - \\
\hline VIII & 1 & & & & & & & & & - & - & - \\
\hline IX & 3 & & & & & & & & & & - & - \\
\hline $\mathrm{X}$ & 1 & & & & & & & & & & & - \\
\hline $\mathrm{XI}$ & 1 & & & & & & & & & & & \\
\hline
\end{tabular}

Table 3. Cosynthetic pattern by precursor feeding method (type strains only).

\begin{tabular}{|c|c|c|c|c|c|c|c|c|}
\hline \multirow{2}{*}{$\begin{array}{l}\text { Convertor } \\
\text { class }\end{array}$} & \multirow{2}{*}{$\begin{array}{l}\text { Type } \\
\text { strain }\end{array}$} & \multicolumn{7}{|c|}{ Secretor class } \\
\hline & & I & III & IV & VI & II & V & $\mathrm{VII}$ \\
\hline I & $4-64$ & - & + & + & + & + & + & + \\
\hline III & $15-52$ & - & - & + & + & + & + & + \\
\hline IV & $18-15$ & - & - & - & + & + & + & + \\
\hline VI & $29-3$ & - & - & - & - & + & + & + \\
\hline II & $13-49$ & - & - & - & - & - & - & - \\
\hline V & $18-67$ & - & - & - & - & - & - & - \\
\hline VII & $30-52$ & - & - & - & - & - & - & - \\
\hline
\end{tabular}

not possible to perform cosynthetic experiments on solid medium because of the production of the unknown antibiotic. The $19 \mathrm{Fur}^{-}$mutants were classified into eleven different groups on the basis of their cosynthetic behavior and biotransformation capability. The results of the cosynthetic experiments are summarized in Tables 2 and 3 . In order to be certain that the antimicrobial activity exhibited in the paper disc diffusion assay was due to furanomycin, all the positive results shown in Table 2 and Table 3 were checked by bioautography. In each case the antimicrobial compound produced by cosynthesis between Fur ${ }^{-}$mutants was found to be chromatographically identical to furanomycin. Each of the cosynthesis experiments 
outlined in Tables 2 and 3 gave the same result when the experiment was repeated four or more times. Some cosynthesis experiments yielded less reproducible results. For example, four repetitions of cosynthesis experiments between the following combinations of mutants gave two positive results and two negative results: $15-65 \times 13-49,15-63 \times 18-67$ and 24-24 × 18-67. Although mutants 15-65, 15-63, 24-24 appear to be in classes, I, II and IV, respectively, they were not selected as type strains because of their erratic behavior, and they were not utilized in precursor feeding experiments. It was observed (Tables 2 and 3 ) that mutants 13-49 (class II), 18-67 (class V) and 30-52 (class VII) exhibited cosynthesis in mixed culture, but not in precursor feeding experiments.

\section{Discussion}

S. threomyceticus Fur $^{-}$mutants were isolated at a significant frequency only after the induction of the mutations by treatment with UV light and NTG in pairwise combination. Under these circumstances, the frequency of Fur $^{-}$mutants was about $0.9 \%$. All mutants had normal morphology (only 22-80 exhibited a change in the spore color) and exhibited normal levels of resistance to furanomycin. This indicated that the mutations did not occur in the genes governing the developmental biology or the self-resistance of $S$. threomyceticus. All mutants except 22-13 (class XI) cosynthesized furanomycin, a result which shows that these mutants are blocked in the biosynthesis pathway to furanomycin. Since mutant 22-13 did not cosynthesize with any other mutants, the mutation in this strain may lie in a regulatory region. Identical behavior has been observed with the act mutants of Streptomyces coelicolor. ${ }^{9}$ On the basis of their cosynthetic and biotransformation behavior, the remaining mutants could be classified into ten distinct phenotypic classes. Seven of these classes could be placed in a linear biosynthetic sequence which proceeds as follows:

$$
\mathrm{I} \rightarrow \mathrm{III} \rightarrow \mathrm{IV} \rightarrow \mathrm{VI} \rightarrow(\mathrm{II}, \mathrm{V}, \mathrm{VII}) \rightarrow \text { furanomycin }
$$

The largest group of mutants fell into the group which served as a convertor for classes II $\sim$ VII, but not as a secretor for any strain. These mutants were assigned to the earliest steps in the biosynthetic sequence and were designated as class $I$. This class of mutants is presumably composed of mutations within a polyketide synthase complex which would be involved in the condensation of propionyl CoA with two successive units of malonyl CoA. Similar classes of mutants have been encountered in the analysis of mutants blocked in the biosynthesis of the polyketides actinorhodin, ${ }^{9)}$ tetracenomycin $\mathrm{C},{ }^{10)}$ and daunomycin. ${ }^{11)}$ Classes II, V and VII could be placed at the end of the linear sequence on the basis of the cosynthetic pattern they exhibited in mixed culture. However, a linear relationship between these three classes could not be established, since they only exhibited cosynthesis in precursor feeding experiments with mutant classes I, III, IV and VI (Table 3). A similar result has been observed with some $\mathrm{Tcm}^{-}$ mutants of Streptomyces glaucescens. ${ }^{10)}$ This behavior may be due to the decomposition of unstable intermediates or to the timing of the feeding experiments. Attempts to observe cosynthesis between mutants 13-49 (class II) and 18-67 (class V) by varying the timing of the precursor feeding experiments were unsuccessful. The remaining three classes of mutant $(\mathrm{VIII} \sim \mathrm{X})$ exhibited cosynthetic behavior which did not allow them to be assigned to any of the other groups nor could they be placed in a linear sequence. The behavior of these three classes of mutant may be due to undetectable levels of cosynthesis, to polar mutations (C. R. Hutchinson; private communication.), or to the presence of more than one mutation in the biosynthetic pathway. Similar behavior is exhibited by some kal mutants of Streptomyces tanashiensis which are blocked in the biosynthetic pathway leading to the polyketide kalafungin. ${ }^{12)}$ As some classes of mutants contained only one member, it is possible that other classes of mutants remain to be isolated. The results of cosynthesis experiments involving some mutants were not reproducible. The reasons for this are unclear. Positive cosynthesis experiments involving class I fur mutants produced levels of furanomycin production that were similar to that of the wild type strain. Positive cosynthesis between other groups of mutants generally exhibited lower levels of production. Three of the mutants employed in these investigations were leaky and occasionally produced low levels of furanomycin. The production of furanomycin by these mutants was always significantly less than that observed in successful cosynthesis 
experiments involving these leaky strains.

The analysis described here of the cosynthetic behavior of the Fur mutants suggests that the biosynthesis of furanomycin proceeds through at least six discrete intermediates after the assembly of the polyketide chain. If the structure of one or more of these intermediates can be elucidated, then this should provide considerable insight into the nature of the steps leading to the formation of the dihydrofuran ring present in furanomycin. Work is in progress to achieve this goal.

\section{Acknowledgment}

We are pleased to acknolwedge financial support of this work by PHS grant no. CA 25142 awarded by the National Cancer Institute, DHHS, and by The Robert A. Welch Foundation (C-729). We thank Professor C. R. HuTCHINSON for helpful comments during the preparation of this manuscript.

\section{References}

1) Katagiri, K.; K. Tori, Y. Kimura, T. Yoshida, T. Nagasaki \& H. Minato: A new antibiotic. Furanomycin, an isoleucine antagonist. J. Med. Chem. 10: 1149 1154, 1967

2) Kohno, T.; D. Kohda, M. Haruk, S. Yokoyama \& T. Mryazawa: Nonprotein amino acid furanomycin, unlike isoleucine in chemical structure, is charged to isoleucine tRNA by isoleucyl-tRNA synthetase and incorporated into protein. J. Biol. Chem. 265: 6931 6935, 1990

3) PARRY, R. J. \& H. P. BuU: Investigations of the biosynthesis of furanomycin. Unexpected derivation from acetate and propionate. J. Am. Chem. Soc. 105: 7446 7447, 1983

4) Parry, R. J.; R. Turakhia \& H. P. BuU: The Biosynthesis of furanomycin: on the mechanism of formation of the ether linkage. J. Am. Chem. Soc. 110: 4035 4036, 1988

5) SCHWAB, J. M. \& J. B. KLASSEN: Steric course of the allylic rearrangement catalyzed by $\beta$-hydroxydecanoylthioester dehydrase. Mechanistic implications. J. Am. Chem. Soc. 106: 7217 7227, 1984

6) CANE, D. E.; T.-C. LiANG \& H. HASLER: Polyether biosynthesis. 2. Origin of the oxygen atoms of monensin A. J. Am. Chem. Soc. 104: 7274 7281, 1982

7) Hopwood, D. M.; M. J. Bibb, K. F. Chater, T. Kieser, C. J. Bruton, H. M. Kieser, D. J. Lydiate, C. P. Smith J. M. WARD \& H. SCHREMPF (Ed.): Genetic Manipulation of Streptomyces. The John Innes Foundation, 1985

8) Delic, V.; D. A. Hopwood \& E. J. FriEND: Mutagenesis by $N$-methyl- $N^{\prime}$-nitro- $N$-nitrosoguanidine (NTG) in Streptomyces coelicolor. Mutation Res. 9: 167 182, 1970

9) RudD, B. A. M. \& D. A. Hopwood: Genetics of actinorhodin biosynthesis by Streptomyces coelicolor A3(2). J. Gen. Microbiol. 114: 35 43, 1979

10) Motamedi, H.; E. Wendt-Pienkowski \& C. R. Hutchinson: Isolation of tetracenomycin C-nonproducing Streptomyces glaucescens mutants. J. Bacteriol. 167: 575 580, 1986

11) Bartel, P. L.; N. C. ConNors \& W. R. STRohl: Biosynthesis of anthracyclines: analysis of mutants of Streptomyces sp. strain C5 blocked in daunomycin biosynthesis. J. Gen. Microbiol. 136: 1877 1886, 1990

12) KaKinuma, S.; H. IKEDA, S. OMURA \& D. A. Hopwood: Biosynthesis of kalafungin in Streptomyces tanashiensis. J. Antibiotics 43: $391 \sim 396,1990$ 